\title{
Regulation Loops for the Ring Magnet Power Supplies in the SSC Accelerator Complex
}

\author{
Eugenio J. Tacconi ${ }^{\dagger}$ and Carlos F. Christiansen ${ }^{\dagger}$ \\ Superconducting Super Collider Laboratory* \\ 2550 Beckleymeade Avenue. MS 4004 \\ Dallas, Texas 75237
}

\section{Abstract}

The SSC complex consists of five cascaded accelerators; the linear accelerator (linac) and four synchrotrons: the low energy booster (LEB), the medium energy booster (MEB), the high energy booster (HEB) and the collider. Twelve or twenty-four pulse phase-controlled SCR power supplies are used for energizing the ring magnets. Each power supply has a voltage loop designed to regulate the voltage applied to the magnets. The voltage regulation loops for these synchrotrons and the current regulation for LEB are analyzed in this work. The digital voltage regulator is fiber-optic isolated from the power converter. It has a closed-loop bandwidth of $150 \mathrm{~Hz}$ with band rejections for $60 \mathrm{~Hz}$ and $120 \mathrm{~Hz}$ perturbations. The LEB has a very precise current regulation system composed by a feedforward compensator, a fast feedback regulator and a slow synchronous regulator. The current regulation design is corroborated by computer simulations.

\section{INTRODUCTION}

The SSC basic design goal is to collide beams of oppositely-directed protons at $20-\mathrm{TeV}$ energy. A cascade of accelerators provides protons at successively higher energy into the following accelerator. Protons are brought to $0.6 \mathrm{GeV}$ in a linear accelerator. Following that are three booster synchrotrons: LEB which accelerates to $11 \mathrm{GeV}$ in a $0.54 \mathrm{~km}$ circumference ring of magnets; MEB, which accelerates to $200 \mathrm{GeV}$ in a $3.96 \mathrm{~km}$ ring; and $\mathrm{HEB}$, which accelerates to 2 $\mathrm{TeV}$ in a $10.9 \mathrm{~km}$ ring. The collider itself consists of two vertically separated rings, each a $20 \mathrm{TeV}$ proton accelerator $87 \mathrm{~km}$ in circumference. The LEB and MEB are synchrotrons using room temperature magnets; while the HEB and collider use superconducting magnets cooled to liquid helium temperature.

The magnet current has to be regulated with a tolerance of a few parts in $10^{6}(\mathrm{ppm})$ in all synchrotrons' magnets. This high current accuracy is obtained by using a fast voltage regulation loop and a slow current regulation system. The dynamic belavior is improved by using feedforward compensation, while adaptive techniques are needed to achieve long term stability. Both, voltage and current regulation systems, are described in the following sections.

\footnotetext{
" Operated by the Universities Research Association, Inc., for the U.S. Department of Energy under Contract No. DEAC35-89ER40486.

$\dagger$ On leave from Leici, UNLP, Conicet, Argentina
}

\section{VOLTAGE REGULATION}

Twelve or twenty-four pulse power supplies are inserted into the ring for energizing the ring magnets. The number and ratings of the power supplies are as follows:

LEB, three twenty-four pulse power supplies, $2000 \mathrm{~V}, 4000 \mathrm{~A}$ MEB, ten twenty-four pulse power supplies, $2000 \mathrm{~V}, 5200 \mathrm{~A}$ HEB, four twenty-four pulse power supplies, $1000 \mathrm{~V}, 7000 \mathrm{~A}$ COLLIDER, forty twelve pulse power supplies, twenty $200 \mathrm{~V}$, $7000 \mathrm{~A}$ and twenty $40 \mathrm{~V}, 7000 \mathrm{~A}$.

Each power supply has its own voltage loop and output passive filter. The voltage loop is designed to have a high frequency response and its main function is to regulate the voltage applied to the magnets. It must follow a predetermined voltage reference related to the current reference, rejecting line voltage changes, offset errors and other voltage perturbations. Due to bandwidth limitations, the voltage loop only rejects voltage perturbations up to $120 \mathrm{~Hz}$ while the output filter rejects the ripple at $360 \mathrm{~Hz}$ and other higher frequency perturbations [1].

The design of the digital voltage regulator is similar in all ring magnet power supplies. The block diagram of the voltage loop is shown in figure 1.

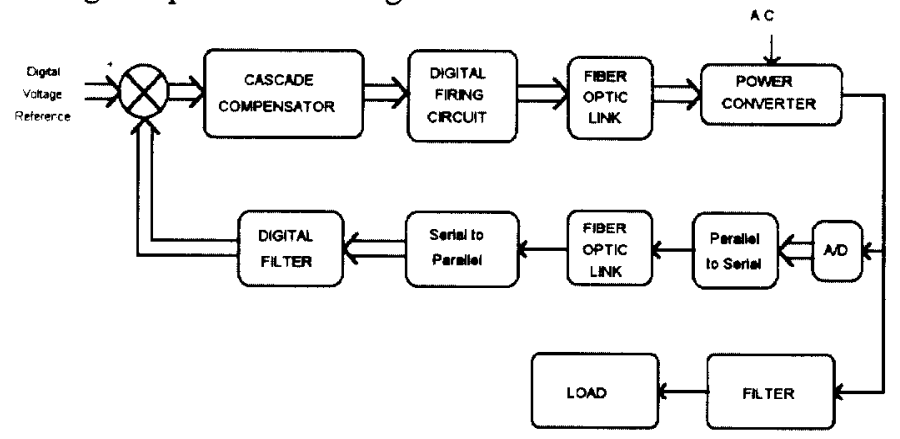

Figure 1, Block Diagram of the Digital Voltage Loop

A distributed grounding system is used in the magnet string. The power supplies voltage to ground changes from one synchrotron to another and is also depending on the synchrotron operating condition. The worst case is in LEB where an electrical isolation of $7.5 \mathrm{kV}$ is required. In order to obtain this high voltage isolation, optical links are used in the voltage regulation for firing the power converter thyristors and to monitor the output voltage (Figure 1). A 12 bit A/D converter digitizes the power converter output voltage at a frequency rate higher than $50 \mathrm{kHz}$. The 12 bits digitized voltage is serial transmitted across the fiber-optic link. The 
optical receiver is followed by a digital filter which calculates the voltage mean value at a frequency rate of $1440 \mathrm{~Hz}$.

The error between voltage reference and voltage feedback goes through a digital cascade compensator that also operates with the $1440 \mathrm{~Hz}$ clock. The compensator has an integral action with very high de gain and two passband filters at 60 $\mathrm{Hz}$ and $120 \mathrm{~Hz}$ respectively. The compensator also includes a linearizer to compensate the non-linear transfer function of the power converter.

The digital firing circuit is basically an open loop system with a single $60 \mathrm{~Hz}$ PLL. It presents a high frequency bandwidth, having a time resolution better than $0.1^{\circ}$.

The voltage loop feature includes a bandwidth of $150 \mathrm{~Hz}$ with more than $20 \mathrm{~dB}$ rejection to $60 \mathrm{~Hz}$ and $120 \mathrm{~Hz}$ perturbations. The ripple at $720 / 1440 \mathrm{~Hz}$ and other higher frequency perturbations are rejected by the output passive filter.

A prototype of the digital voltage regulator has been developed and tested, with good results, in a twelve pulse phase-controlled SCR power supply.

\section{CURRENT REGULATION}

The period of the magnet current waveforms ranges from 0.1 second for LEB up to about 24 hours for collider. A current regulation tolerance of a few parts in $10^{6}$ is required in all synchrotron magnets and in some cases (MEB Collider) a precise tracking between bending magnets and quadrupoles or between sectors is also required. In order to satisfy these requirements, different regulation schemes, including feedforward, feedback and adaptive techniques are used. In fast cycling synchrotrons (LEB, MEB) the emphasis is made in obtaining a good dynamic behavior, while in the slower superconducting magnets (HEB, Collider) the main current regulation problems are related to long term stability and noise and ripple reduction. Among the different ring magnet current regulation loops, only the current regulation design for LEB $10 \mathrm{~Hz}$ biased sinewave operation mode is described in this paper.

The LEB magnet system consists of 48 dipoles and 90 quadrupoles connected in series circuit. The power system has to operate in either of two modes: $10 \mathrm{~Hz}$ biased sine wave and linear ramp. For $10 \mathrm{~Hz}$ biased sinewave operation, dipoles and quadrupoles magnets resonate with 12 distributed capacitors [2]-[4]. The capacitors are bypassed by a $40 \mathrm{mHy}$ choke to provide a path for the $d-c$ component of the magnet current. For the linear operation mode, the twelve resonant banks are short-circuited. Three 24-pulse SCR phasecontrolled power supplies are inserted into the ring to produce both current waveforms.

The maximum and minimum current flowing through the magnets has to be regulated, in both modes, within $50 \mathrm{ppm}$ of the actual current. A typical required magnet current waveform for the $10 \mathrm{~Hz}$ biased sinewave mode is shown in figure 2 . For satisfying this requirement, a current regulation system with a high open-loop gain at $\mathrm{dc}$ and $10 \mathrm{~Hz}$ is necessary.

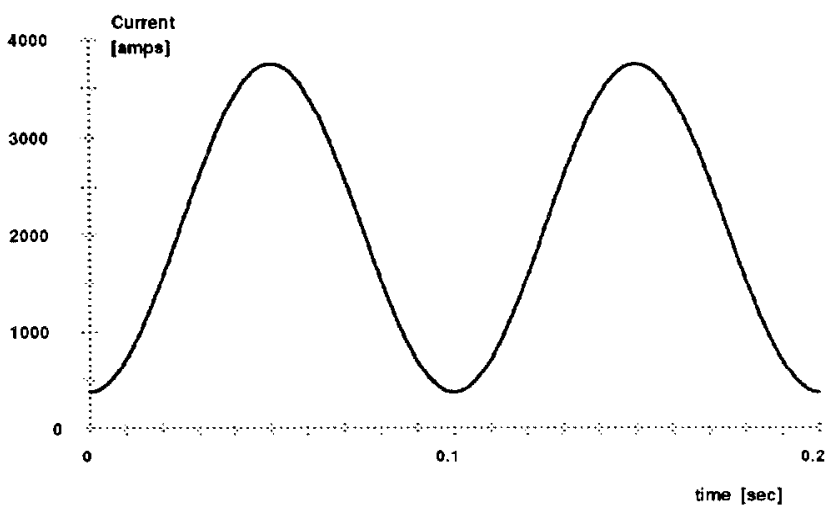

Figure 2. Magnet Current Waveform $10 \mathrm{~Hz}$ Biased Sinewave mode

A high open-loop gain at $10 \mathrm{~Hz}$ with adequate closedloop stability can not be simultaneously fulfilled by using a conventional regulator. The regulation problem has been solved by combining feed-forward compensation and conventional feedback regulation with frequency conversion techniques.

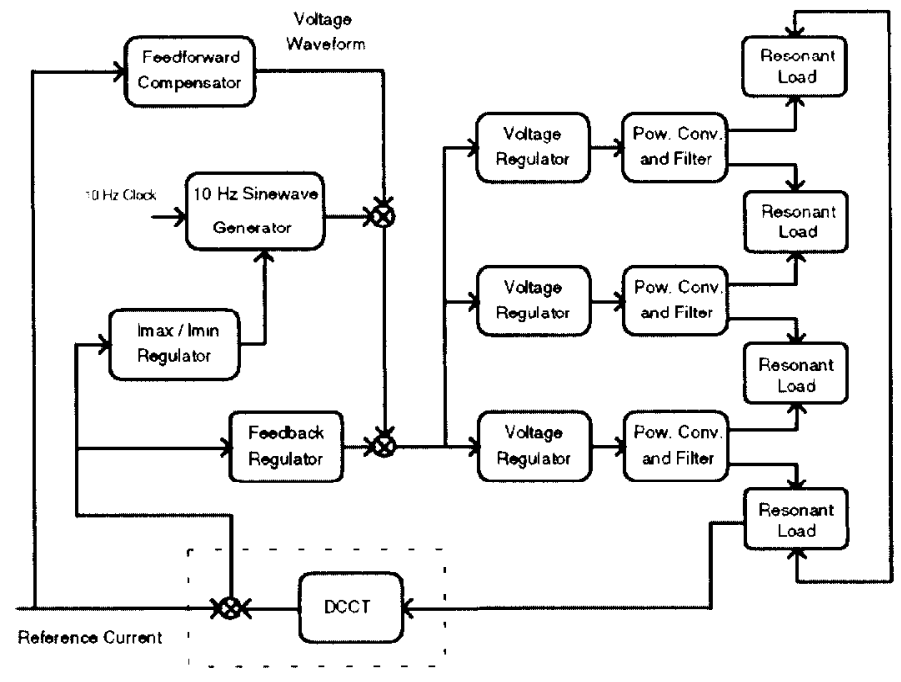

Fig. 3. Block diagram of the current loop $10 \mathrm{~Hz}$. Biased Sinewave mode

The schematic diagram of the current loop is shown in figure 3. The central computer provides the maximum and minimum current values while the $10 \mathrm{~Hz}$ biased sinewave current reference, synchronized with the utilities, is locally generated.

The magnet current is measured with a very precise Zero-Flux DC Current Transducer (DCCT). An analog differential amplifier with a gain of 30 amplifies the difference between the current reference and the magnet 
current. A 18 bit D/A converts the digital reference current while a 16 bit A/D digitizes the differential amplifier output. $\mathrm{D} / \mathrm{A}, \mathrm{A} / \mathrm{D}$ converters and the differential amplifier are built inside the DCCT in a temperature regulated chamber with a precision of $0.01^{\circ}$. The same current measuring system is used for both LEB operation modes; while at least two high precision DCCTs were required in a former current regulation design [5].

The digital current regulator is fully digital operating at a frequency rate of $1440 \mathrm{~Hz}$. It is composed by a feedforward compensator, a feedback regulator and a synchronous $\mathrm{I}_{\max } / \mathrm{I}_{\min }$ regulator. The voltage reference is generated by adding the outputs of the three regulation blocks. The three ring magnet power supplies are driven by the same voltage reference.

\section{Feedforward Compensator}

The feedforward compensator provides the necessary voltage for having a current flowing through the magnets that equals the reference current. This voltage waveform is also a biased sine wave calculated considering the load in perfect resonance. A feedforward system is an open-loop compensation unable to correct current perturbations. It has to be usually complemented with a feedback system.

\section{Feedback Regulator}

For designing the feedback loop, the load characteristic has to be fully understood. The voltage loop has a bandwidth of $150 \mathrm{~Hz}$ and the output passive filter is basically a second order, critically damped, low-pass filter with a resonant frequency of about $130 \mathrm{~Hz}$. The admittance of the resonant load is represented in frequency domain in figure 4.

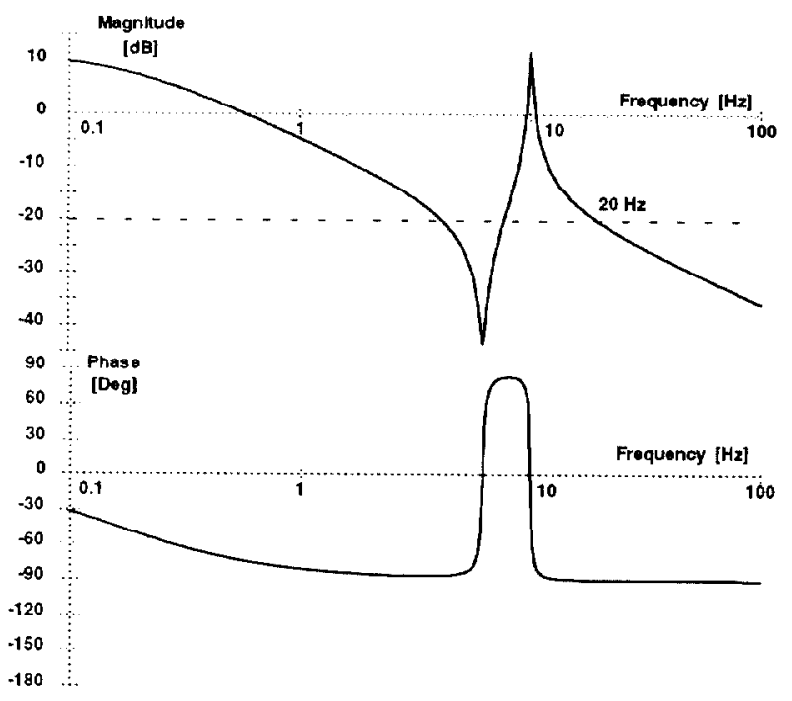

Figure 4. Magnitude and phase frequency response of the load admittance
The feedback regulator has been designed with a proportional cascade compensator with a total gain of $20 \mathrm{~dB}$. This additional gain is indicated in dotted line in figure 4. The feedback loop is stable, having a closed-loop bandwidth of $20 \mathrm{~Hz}$. Considering that the phase introduced at $20 \mathrm{~Hz}$ by the voltage loop and passive filter is less than $30^{\circ}$, a $60^{\circ}$ phase margin is obtained. The feedback loop has a gain of 30 $\mathrm{dB}$ at $\mathrm{dc}$ and $10 \mathrm{~Hz}$. This gain is not enough to satisfy the regulation requirements. The gain at low frequencies can be increased using integral action but the gain at $10 \mathrm{~Hz}$ cannot be substantially increased, using standard techniques, without reducing the phase stability margin.

\section{Synchronous Regulator}

The basic idea of the synchronous regulator is to take advantage of the periodicity of the reference current. The current error is measured during one signal pcriod and the correction is synchronously and gradually applied during the following periods. In this way, an equivalent high gain at 10 $\mathrm{Hz}$ can be obtained while limiting the closed-loop bandwidth of the synchronous regulator to less than $1 \mathrm{~Hz}$.

The high gain in the low frequency band is easily obtained by integrating the mean value of the error signal (dc). The integrator gain has been calculated for having a correction bandwidth of $0.6 \mathrm{~Hz}$.

Frequency conversion techniques are used for obtaining high gain at $10 \mathrm{~Hz}$. The residual $10 \mathrm{~Hz}$ sine wave on the error signal is detected in amplitude (ac component only) and integrated. The output of this integrating amplifier modulates the amplitude of a synchronized sine wave.

The first step to design the a-c amplitude regulation loop is to obtain a mathematical model of the system including detection and modulation processes. The block diagram of figure 5 will be used for this purpose.

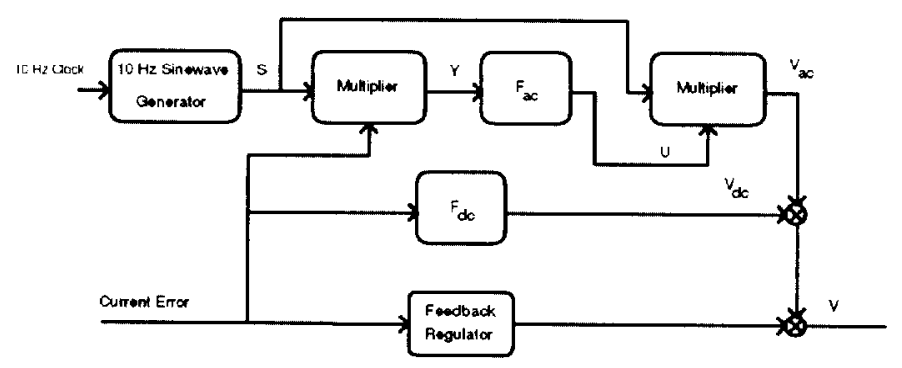

\section{Fig. 5. Block diagram representing the detection and modulation process}

In the above figure, the signal demodulation has been implemented with a synchronous or a linear phase detector [6]. In fact, as the carrier frequency is available, this efficient method for amplitude or phase detection can be readily implemented. The ac and dc components of the error signal are filtered with the integrating amplifiers $F_{a c}$ and $F_{d c}$ respectively. 


$$
F_{a c}=\frac{K_{a c}}{s} \quad F_{d c}=\frac{K_{d c}}{s}
$$

The amplitude modulation and deteclion are nonlinear processes that can be handled by the Laplace transform using s-plane convolution [7]. The same structure employing a multiplier excited by the $10 \mathrm{~Hz}$ sine wave is used in figure 5 for implementing both, modulation and detection processes.

Denoting the Laplace transform of $\mathrm{e}_{\mathrm{ac}}(\mathrm{t})$ and $\mathrm{u}(\mathrm{t})$ by $\mathrm{E}_{\mathrm{ac}}(\mathrm{s})$ and $\mathrm{U}(\mathrm{s})$, the transform of both products can be expressed by the following s-plane convolutions.

$$
\begin{aligned}
& U(s)=\frac{1}{2 \pi j} \int S(\lambda) \cdot E_{a c}(s-\lambda) \cdot d \lambda \\
& V_{a c}(s)=\frac{1}{2 \pi j} \int S(\lambda) \cdot U(s-\lambda) \cdot d \lambda
\end{aligned}
$$

Both convolution integrals are readily evaluated by taking residues at poles of $S(\lambda)$. Neglecting high frequency components, the transfer function of the ac synchronous regulator can be approximated by

$$
\frac{V_{a c}(s)}{E(s)} \cong \frac{K_{a c}}{2} \cdot \frac{s}{s^{2}+\omega_{0}^{2}}
$$

Thus, the transfer function of the equivalent feedhack regulator can be expressed by

$$
\frac{V(s)}{E(s)}=K_{p}+\frac{K_{d c}}{s}+\frac{K_{a c}}{2} \cdot \frac{s}{s^{2}+\omega_{o}^{2}}
$$

where $K_{p}$ represents the proportional gain of the feedback regulator.

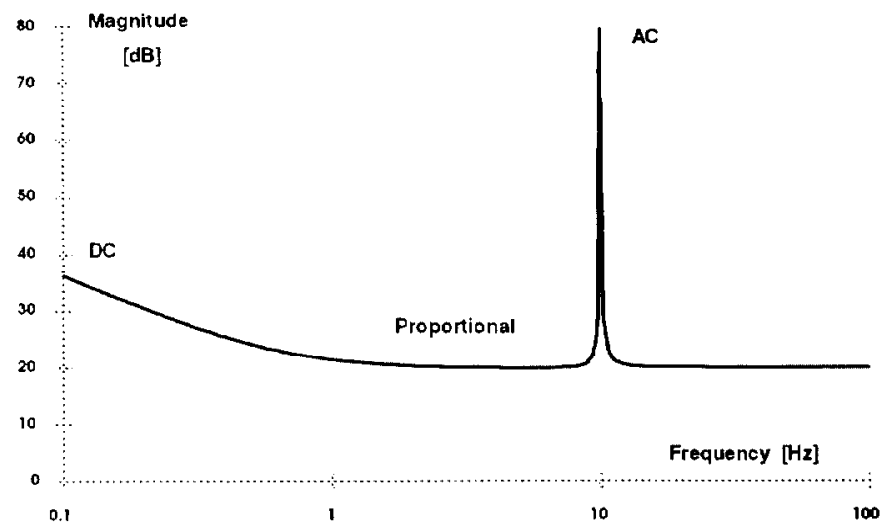

Figure 6. Magnitude frequency response of the equivalent feedback regulator
The frequency response of the equivalent feedback regulator is represented in figure 6 using the following constant values: $\mathrm{K}_{\mathrm{p}}=10, \mathrm{~K}_{\mathrm{dc}}=40, \mathrm{~K}_{\mathrm{ac}}=160$. The effect of the proportional feedback regulator as well as the ac and $\mathrm{dc}$ components of the synchronous regulator can be easily identify in figure 6 . Using equation (5), the transfer function of the total open-loop feedback regulation can be calculated. Its frequency response is represented in figure 7.

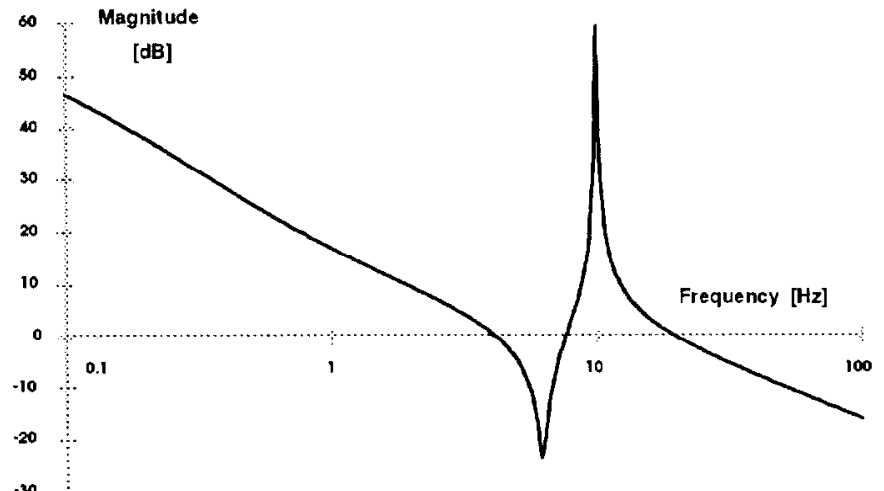

Figure 7. Magnitude frequency response of the total open-loop feedback regulation

Comparing figures 4 and 7, the improvement of the synchronous regulator is apparent. The $20 \mathrm{~Hz}$ closed-loop bandwidth, controlled by the proportional constant $\mathrm{K}_{\mathrm{p}}$, has not been modified. The system is stable, the phase margin has been reduced in only $6^{\circ}$ (equation (5)) and the equivalent open-loop gain at $\mathrm{dc}$ and $10 \mathrm{~Hz}$ has been dramatically increased. The constant values $\mathrm{K}_{\mathrm{dc}}$ and $\mathrm{K}_{\mathrm{ac}}$ have been chosen for having a correction bandwidth of $0.6 \mathrm{~Hz}$ for both, $\mathrm{dc}$ and $10 \mathrm{H} z$ components.

\section{SIMULATION RESULTS}

The linear phase detector of figure 5 has been replaced, in figure 3 and in the computer simulations, by a timing circuit and two sample and hold. The timing circuit generates sample pulses synchronized with the maximum and minimum of the magnet current. Two sample-and-hold circuits measure the current error at the maximum and minimum points. Thus, this measuring system works in a similar way as a synchronous detector but has a different d-c gain. In fact, if the crror signal is a non-biased sinusoid with amplitude $\hat{I}$ and the same frequency and phase of the carrier signal $s(t)$, the difference between maximum and minimum sample-and-hold outputs will yield a d-c component of amplitude $2 \hat{I}$. Under similar conditions, the output of a linear phase detector would be $\hat{I} / 2$. The addition of the maximum and minimum sample-and-hold outputs is proportional to the error signal bias and is used to drive the $\mathrm{dc}$ regulator. The dynamic behavior of the feedforward compensation and the 
feedback regulation is shown in the simulation results of figures 8 and 9 .

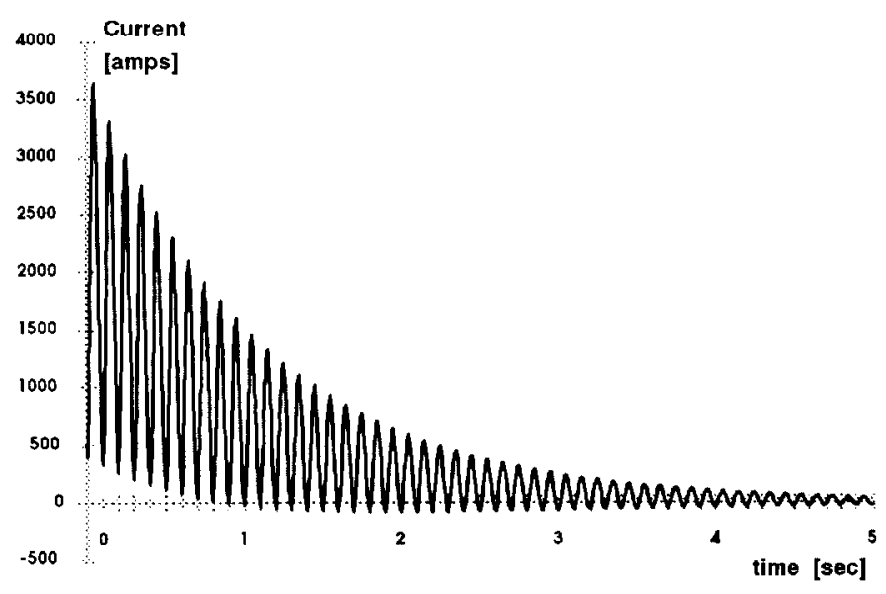

Figure 8. Difference between reference and magnet current by using feedforward compensator

The time domain response of the feedforward compensator is shown in figure 8 . At the time origin, the feedforward compensator is connected while the feedback and the synchronous regulator remain disconnected. The reference current waveform is as shown in figure 2 . The figure shows that if a good model of the plant is available, due to the action of the feedforward compensator, the current error goes to zero in a few seconds.

Due to changes in the load, specially in the resonant capacitors, a good model of the load is not always available. In the following simulation the feedforward compensator output has a $10 \%$ error in the dc value as well as in the sinewave amplitude. The feedback regulator has a fast response ( $20 \mathrm{~Hz}$ bandwidth) and reduces the residual $\mathrm{dc}$ and ac current errors by a factor of 30 .

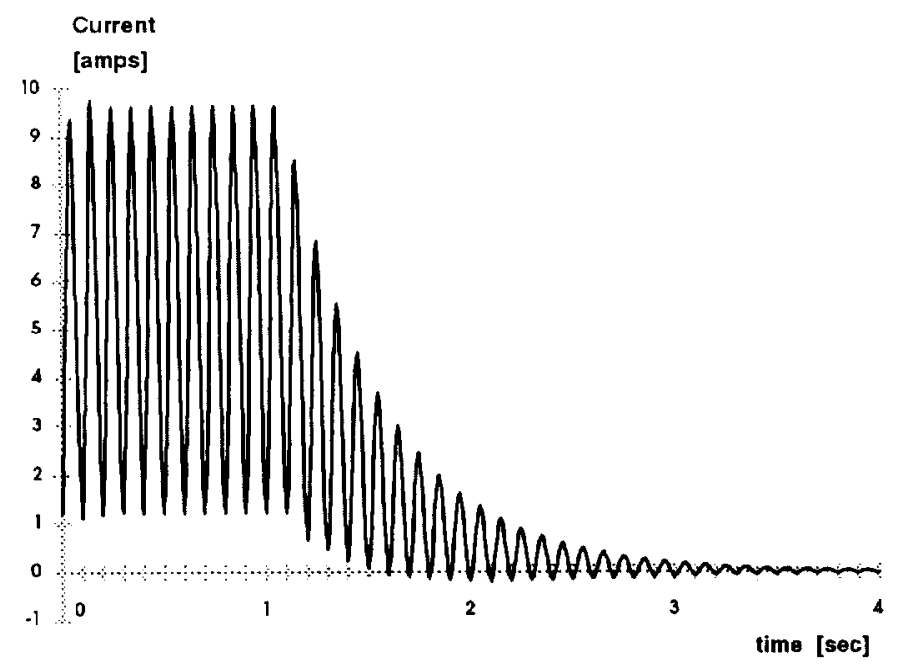

Figure 9. Difference between reference and magnet current by using feedback synchronous regulator
In the first part of figure 9, a $10 \%$ error feedforward compensator and the fecdback regulator are in steady-state. At a time of $1 \mathrm{sec}$, the ac/dc synchronous regulator is connected. The figure shows that the residual current error goes to zero with a constant time, for both ac/dc components, of about $0.25 \mathrm{sec}$, corresponding to a bandwidth of $0.6 \mathrm{~Hz}$.

\section{CONCLUSIONS}

The analysis and design of the digital voltage regulation for all ring magnet power supply as well as the current regulation system for biased sinewave current mode of LEB have been presented.

Conventional feedforward and feedback techniques are not enough to maintain the magnet current within the required tolerance. A synchronous feedback regulator based on the periodicity of the current waveform has been used to improve the current regulation performance. The current regulation design has been corroborated by computer simulations. The prototype of the described digital current regulator is still under development.

\section{REFERENCES}

[1]E. Tacconi. "LEB Ring Magnet Power Supply System, Voltage and Current Regulation Design", SSC Laboratory SSCL-N-785, January (1992).

[2] J. R. Sanford and D. M. Matthews, "Site-Specific Conceptual Design of the Superconducting Super Collider", SSC Laboratory, SSCL-SR-1056, July (1990).

[3] C. Jach, "Switchable $10 \mathrm{~Hz} / 1 \mathrm{~Hz}$ LEB Magnet Power Supply System", Conference Record of the 1991 IEEE Particle Accelerator Conference, Vol. 2, pp. 816-818.

[4] C. Jach, R. Winje, E. Tacconi, R. Harold and K. Smedley. "Low Energy Boolster Main Magnet Power Supply System", Proceedings of the 1992 Third European Particle Accelerator Conference, Vol. 2, pp. 1438-1440.

[5] E. Tacconi, C. Jach and R. Winje. "10 Hz resonant LEB magnet power supply system, current regulation design", Conference Record of 1992 IEEE Nuclear Science Symposium and Medical Imaging Conference, Vol. 2, pp. 1018-1022.

[6]P. Horowitz and W. Hill, "The art of Electronics", Cambridge University Press, (1989).

[7] W. R. LePage, "Complex Variables and the Laplace Transform for Engineers", New York, Dover Publications, (1980). 